\title{
Molecular Basis of Spectrin Deficiency in $\beta$ Spectrin Durham \\ A Deletion within $\beta$ Spectrin Adjacent to the Ankyrin-binding Site Precludes Spectrin Attachment to the Membrane in Hereditary Spherocytosis
}

\author{
Hani Hassoun, ${ }^{*}$ John N. Vassiliadis, ${ }^{\star}$ James Murray, ${ }^{\star}$ Scott J. Yi, ${ }^{\star}$ Manjit Hanspal, ${ }^{\star}$ Russell E. Ware, ${ }^{\ddagger}$ Stuart S. Winter, ${ }^{\ddagger}$ \\ Shyh-Shin Chiou, ${ }^{*}$ and Jiri Palek* \\ * Department of Biomedical Research and the Division of Hematology/Oncology, St. Elizabeth's Hospital of Boston, Tufts University \\ Medical School, Boston, Massachusetts 02135; and ${ }^{\ddagger}$ Department of Pediatrics, Duke University Medical Center, Durham, \\ North Carolina 27710
}

\begin{abstract}
We describe a spectrin variant characterized by a truncated $\beta$ chain and associated with hereditary spherocytosis. The clinical phenotype consists of a moderate hemolytic anemia with striking spherocytosis and mild spiculation of the red cells. We describe the biochemical characteristics of this truncated protein which constitutes only $10 \%$ of the total $\beta$ spectrin present on the membrane, resulting in spectrin deficiency. Analysis of reticulocyte cDNA revealed the deletion of exons 22 and 23. We show, using Southern blot analysis, that this truncation results from a 4.6-kb genomic deletion. To elucidate the basis for the decreased amount of the truncated protein on the membrane and the overall spectrin deficiency, we show that $(a)$ the mutated gene is efficiently transcribed and its mRNA abundant in reticulocytes, $(b)$ the mutant protein is normally synthesized in erythroid progenitor cells, $(c)$ the stability of the mutant protein in the cytoplasm of erythroblasts parallels that of the normal $\beta$ spectrin, and $(d)$ the abnormal protein is inefficiently incorporated into the membrane of erythroblasts. We conclude that the truncation within the $\beta$ spectrin leads to inefficient incorporation of the mutant protein into the skeleton despite its normal synthesis and stability. We postulate that this misincorporation results from conformational changes of the $\beta$ spectrin subunit affecting the binding of the abnormal heterodimer to ankyrin, and we provide evidence based on binding assays of recombinant synthetic peptides to insideout-vesicles to support this model. (J. Clin. Invest. 1995. 96:2623-2629.) Key words: hereditary spherocytosis $-\beta$ spectrin • deletion • erythrocyte membrane $\cdot$ cytoskeleton
\end{abstract}

\section{Introduction}

The erythrocyte skeleton that laminates the inner side of the red cell membrane is formed by the noncovalent interaction between four major proteins: spectrin, actin, protein 4.1 , and protein 4.9 (reviewed in references 1,2 ). Spectrin, the most

Address correspondence to Hani Hassoun, Division of Hematology/ Oncology, St. Elizabeth's Hospital, 736 Cambridge Street, Boston, MA 02135. Phone: 617-789-5018; Fax: 617-254-7488.

Received for publication 14 October 1994 and accepted in revised form 10 August 1995.

J. Clin. Invest.

(C) The American Society for Clinical Investigation, Inc.

0021-9738/95/12/2623/07 \$2.00

Volume 96, December 1995, 2623-2629 abundant skeletal protein, consists of two chains, $\alpha$ and $\beta$, intertwined in an antiparallel manner to form heterodimers ( 3 , 4 ) which associate at their head region to form tetramers ( 5 , 6 ). This skeleton is attached to the membrane mainly by ankyrin which connects spectrin to the major transmembrane protein, band 3. This skeletal network plays an essential role in determining the shape and deformability of the red cell (reviewed in reference 1).

In recent years, mutations leading to deficiency or dysfunction of these membrane proteins have been associated with hereditary hemolytic anemias. Different phenotypes have been described in association with defects of specific proteins or specific functional domains. Hereditary spherocytosis (HS $)^{1}$ and elliptocytosis are the two red cell phenotypes best studied to date.

HS is a common hemolytic anemia (reviewed in reference 1) with a broad spectrum of clinical severity. Two forms of the disease have been described: a dominant form and a less common but more severe recessive form $(7,8)$. Red cells from HS patients exhibit varying degrees of surface area deficiency leading to spherocytosis and increased red cell osmotic fragility. Defects of several red cell membrane proteins have been associated with the disease. Mirroring the clinical heterogeneity, several subsets of patients have been described based on SDSPAGE or RIA analysis of the red cell membrane proteins: isolated spectrin deficiency (7-9), combined spectrin and ankyrin deficiency $(10-12)$, band 3 deficiency $(13-16)$, and defects of protein $4.2(17-20)$ have all been associated with hereditary spherocytosis. The correlation between these different subsets and the clinical expression is currently not well established.

Recently, the underlying molecular defects have been elucidated in some cases of hereditary spherocytosis: mutations involving band 3 have been described in patients with HS associated with band 3 deficiency $(20,21)$. Likewise, mutations involving the ankyrin gene have been uncovered in patients with combined spectrin and ankyrin deficiencies $(22,23)$. In the majority of patients, these defects are expressed in the heterozygous state. Regarding spectrin, a point mutation of the $\beta$ spectrin gene causing a defective binding of spectrin to protein 4.1 has been found in a family with dominantly inherited HS associated with isolated spectrin deficiency $(24,25)$. Based on in vitro peptide expression assays, instability and proteolytic degradation of this mutated protein was presumed to be the basis for the spectrin deficiency. So far, no mutations of $\alpha$ spectrin have been reported. This is not surprising knowing that the $\beta$ spectrin

1. Abbreviations used in this paper: HS, hereditary spherocytosis; IOVs, inside-out-vesicles. 
synthesis rate is the limiting factor for spectrin assembly on the membrane, as the synthesis of $\alpha$ spectrin has been shown to be threefold that of $\beta$ spectrin $(26,27)$. Therefore, in contrast to $\alpha$ spectrin, defects of $\beta$ spectrin are more likely to be expressed in the heterozygous state.

We have studied a truncated $\beta$ spectrin associated with a phenotype of HS with overall spectrin deficiency. Southern blot analysis of the patient's genomic DNA revealed that this truncation is the result of a large genomic deletion causing an inframe deletion of two exons at the transcriptional level. Because the mutant protein constitutes only $10 \%$ of the total $\beta$ spectrin present on the membrane, we conducted biosynthetic studies to explain the decrease in the amount of the truncated protein and the overall spectrin deficiency. We show that the mutant gene is normally transcribed and its message is stable in reticulocytes and that the synthesis and stability of the mutant protein is the same as that of its normal counterpart in the cytoplasm of erythroid progenitors. However, we show that, despite its normal synthesis and stability, the abnormal $\beta$ spectrin is inadequately incorporated into the membrane of erythroblasts. Because the deletion involves repeated segments 12 and 13 located near the ankyrin-binding site, we propose that conformational changes of the protein lead to a defective binding of the mutant spectrin to ankyrin, thereby reducing the recruitment of $\alpha$ spectrin$\beta$ spectrin heterodimers containing the mutant spectrin to the membrane. We provide data, based on binding of recombinant synthetic peptides to inside-out-vesicles (IOVs), to corroborate this model.

\section{Methods}

Patient. The family under study consists of three members: the mother and the father are asymptomatic and present no detectable hematologic abnormalities. Their daughter initially presented at age 7 with an aplastic crisis with a hemoglobin of $4.4 \mathrm{~g} / \mathrm{dl}$ and a reticulocyte count of 2 . The physical examination revealed splenomegaly. After recovery from this acute event, her hemoglobin remained around $10-11 \mathrm{mg} / \mathrm{dl}$, with a mean corpuscular volume of 82 , a reticulocyte count between 10 and $20 \%$, a bilirubin $\sim 1.8 \mathrm{mg} / \mathrm{dl}$, and a lactate dehydrogenase level around $900 \mathrm{IU} /$ liter. Her peripheral blood smear shows marked spherocytosis with moderate spiculation, and her red cell osmotic fragility is markedly increased.

Erythrocyte membrane protein analysis. The methods used for analysis of the erythrocyte membrane proteins have been described previously and include $(a)$ erythrocyte membrane preparation $(28,29)$; (b) analysis of the red cell membrane proteins by SDS-PAGE (3.5$17 \%$ gradient Fairbanks gels and $10 \%$ Laemmli polyacrylamide gels) (30); (c) immunoblotting using polyclonal antibodies raised against $\alpha$ spectrin, $\beta$ spectrin, and ankyrin $(28) ;(d)$ spectrin extraction at $4^{\circ} \mathrm{C}$ (28) and relative determination of spectrin dimers and spectrin tetramers by nondenaturing gel electrophoresis $(31)$; and $(e)$ second dimension SDS-PAGE of spectrin dimers and spectrin tetramers initially separated by nondenaturing gel electrophoresis at $4^{\circ} \mathrm{C}$. For this assay, the spectrin dimer and tetramer bands were cut off the nondenaturing tube gels and run on $10 \%$ polyacrylamide Laemmli gels.

PCR amplification of reticulocyte $\beta$ spectrin cDNA. $5 \mu \mathrm{g}$ of total RNA isolated from reticulocytes (32) were reverse transcribed using random primers. The $\beta$ spectrin cDNA segments extending from exons 21-25 and exons 21-26 were amplified by the PCR using two sets of primers respectively: sense primer K11 on exon 21 ( $5^{\prime}$-TGGACCTCCTGGAACCCCTA-3') with antisense primer 352 on exon 25 ( $5^{\prime}$-ACATGTTCTCCAGCTTGCG-3') and sense primer 296 on exon 21 (5'TCCAGAGCCAAGCTGCAGAT-3') with antisense primer 297 on exon 26 ( $5^{\prime}$-TCCTTCCACTCGGCGATGGT-3') shown in Fig. 2. The fragments resulting from this amplification were fractionated on a $1 \%$ agarose gel and stained with ethidium bromide.

Southern analysis of genomic DNA. Probes were labeled with $\left[{ }^{32} \mathrm{P}\right]-$ dATP by random priming (Megaprime DNA labeling system; Amersham International, Little Chalfont, UK). Genomic DNA was isolated from PBMCs as described previously (33). $10 \mu \mathrm{g}$ of genomic DNA was digested with two restriction enzymes independently (XbaI and EcoRI). The resulting fragments were fractionated on a $0.7 \%$ agarose gel, transferred to a nylon membrane (Zetaprobe; Bio-Rad Laboratories, Inc., Richmond, CA) by nonalkaline Southern blotting, and hybridized to the probe as described (34).

PCR amplification of $\beta$ spectrin genomic DNA. Genomic DNA was isolated from peripheral blood leukocytes as described previously (33). Two sets of primers were used independently: sense primer $\mathrm{K} 11$ on exon 21 ( $5^{\prime}$-TGGACCTCCTGGAACCCCTA-3') with antisense primer 300 on exon 24 ( $5^{\prime}$-CCGCAAATGTCGCTTCAGCA-3') and sense primer 296 on exon 21 ( $5^{\prime}$-TCCAGAGCCAAGCTGCAGAT-3') with antisense primer 300 on exon 24 (Fig. 3).

Subcloning and sequencing of amplified DNA. DNA fragments generated by PCR amplification were fractionated on a $1 \%$ agarose gel, purified using the Geneclean system (Bio 101, La Jolla, CA), and subcloned into the pCRII cloning vector (Invitrogen Corp., San Diego, CA). After transformation in competent Escherichia coli cells, the recombinant DNA clones were sequenced by the dideoxynucleotide sequencing method of Sanger et al. (35).

cDNA quantitation by PCR. mRNA was prepared from peripheral blood reticulocytes and reverse transcribed to cDNA using random primers. To quantify the relative amounts of the normal and abnormal transcripts, we amplified by PCR a cDNA segment overlapping the truncated fragment using primers 296 and 297 located on exons 21 and 26, respectively (data not shown). This amplification results in an 888-bp DNA fragment from the normal allele, and a 519-bp fragment from the truncated allele. A $500-\mu \mathrm{l}$ reaction mixture containing $50 \mathrm{mM} \mathrm{KCl}, 10 \mathrm{mM}$ Tris- $\mathrm{HCl}, 2 \mathrm{mM} \mathrm{MgCl}_{2}, 0.1 \%$ gelatin, $100 \mathrm{pmol}$ of each primer, 0.2 $\mathrm{mM}$ radioactive dATP, $0.2 \mathrm{mM}$ of the other $\mathrm{dNTP}$, and $2 \mathrm{U}$ of Taq polymerase was aliquoted in 15 PCR tubes. A 30-cycle PCR amplification was initiated. The tubes were removed successively at the end of each cycle between cycles 15 and 30 . The DNA fragments resulting from this amplification were fractionated on a $5 \%$ polyacrylamide gel overnight at $180 \mathrm{~V}$, and their amount quantified by exposure of the gel to autoradiography followed by densitometric analysis of the film. Also, after localization by autoradiography, the bands were cut off the gel, and the radioactive signal was quantified by beta spectrometer. The relative abundance of the truncated band to the normal band was determined for each one of the 15 samples.

In vitro assay of spectrin synthesis, stability, and incorporation into the membrane. The methods used for this assay have been extensively described elsewhere (36). Briefly, they include $(a)$ a two-phase liquid culture system for the maturation of erythroid progenitors using the system described by Fibach et al. (37) and (b) pulse label and pulse chase experiments of late erythroblasts isolated from liquid culture followed by immunoprecipitation of spectrin from the cytoplasmic and skeletal fractions using affinity-purified antispectrin antibodies by the method described previously (36).

Bacterial expression of recombinant proteins. cDNA was prepared from the patient's reticulocytes. Using sense primers E1 (5'-GCTGGATCCATGGAGGACCAGCTGCGG-3') and antisense primer E $\overline{2\left(5^{\prime}-\right.}$ CGCGAATTCCTGGGTCCGGCGCCCGGC-3') attached to linkers containing BamHI and EcoRI restriction sites, respectively (underlined), fragments overlapping the deleted region were amplified by the PCR. Two fragments corresponding to the normal and the truncated mutant alleles were subcloned into pGEX-2T. After transformation into strain DH5 $\alpha$ E. coli, the expression of the recombinant peptides was induced by isopropyl- $\beta$-D-thiogalactopyranoside, and the normal and truncated peptides were recovered separately from bacterial lysates using glutathione agarose beads as described (38). The two synthesized peptides extend from residues 1397 to 1898 of the $\beta$ spectrin protein, therefore containing the ankyrin-binding domain of spectrin. The choice 
of these specific peptides (i.e., from residues 1397 to 1898) was based on the fact that the binding of the normal peptide to IOVs has been extensively studied and reported previously (39).

Erythrocyte IOVs binding assays. Spectrin- and actin-depleted IOVs were prepared as described previously (40). Spectrin dimers extracted from erythrocyte ghosts were purified on sucrose gradient as described elsewhere (41) and iodinated as recommended by the manufacturer (Bolton-hunter reagent ${ }^{125}$ I kit; ICN Radiochemicals Div., ICN Biochemicals Inc., Irvine, CA). The competitive inhibition of binding of iodinated spectrin to IOVs by each of the two synthetic peptides was explored and compared as described by Van Bennett and Branton with minor modifications (41). In brief, $15 \mu \mathrm{g}$ of IOVs and a fixed amount of radiolabeled spectrin $(7 \mu \mathrm{g})$ were incubated for $90 \mathrm{~min}$ with increasing amounts of each peptide $(0.05-20 \mu \mathrm{g})$ in $200 \mu \mathrm{l}$ of $10 \mathrm{mM} \mathrm{Na}_{2} \mathrm{HPO}_{4}$, $150 \mathrm{mM} \mathrm{NaCl}, 1 \mathrm{mM}$ EDTA, and $1 \mathrm{mM}$ DTT, pH 7.4. After incubation, the solutions were layered on $0.2 \mathrm{ml}$ of $20 \%$ sucrose in polyethylene microfuge tubes, and the peptide-bound IOVs recovered in the pellet after centrifugation, at $18,000 \mathrm{rpm}$ for $30 \mathrm{~min}$. The microfuge tubes were then frozen in liquid nitrogen, and the tips containing the pelleted IOVs were sectioned and their radioactivity measured to estimate the amount of iodinated spectrin bound.

\section{Results}

\section{Biochemical characterization of the mutant $\beta$ spectrin}

Analysis of the patient's red cell membrane proteins by $3.5-$ $17 \%$ gradient SDS-PAGE revealed an additional band migrating close to band 2.1 (data not shown). When analyzed on a $10 \%$ Laemmli gel, this extra band could be clearly separated from the ankyrin/spectrin band. (Fig. $1 A$ ). Western blot analysis using polyclonal antibodies raised against $\alpha$ spectrin, $\beta$ spectrin, and ankyrin revealed the $\beta$ spectrin origin of this extra band (Fig. $1 B$ ). The apparent molecular mass of this truncated $\beta$ spectrin protein was $205 \mathrm{kD}$ and constituted $\sim 10 \%$ of the $\beta$ spectrin present on the membrane. Quantitation of the total amount of spectrin on the membrane (including the truncated spectrin) using densitometric analysis of SDS-PAGE gels and expressed as the ratio of spectrin to band 3 revealed a significant decrease in spectrin (spectrin to band 3 ratio of 0.84 , normal: $0.97 \pm 0.10$ ). This spectrin deficiency is even more pronounced when the truncated spectrin is excluded (spectrin/b3 of 0.80 ). Both parents had a normal amount of spectrin on the membrane as assessed by this method. Nondenaturing gel electrophoresis of spectrin extracts performed at $4^{\circ} \mathrm{C}$ showed a highly reproducible increase in the percentage of spectrin dimers out of the total pool of spectrin (i.e., spectrin dimers and spectrin tetramers) averaging $18 \%$ (normal being $<10 \%$ ) (Fig. $1 C$ ). The two dimensional analysis by SDS-PAGE of spectrin dimers and spectrin tetramers showed the dimeric fraction to be enriched in the truncated protein (Fig. 1 D).

\section{Characterization of the molecular defect underlying the truncated $\beta$ spectrin}

After preparing cDNA from the patient's reticulocytes, PCR amplification of overlapping fragments spanning the entire $\beta$ spectrin cDNA was carried out. Amplification of the sequence derived from exons $21-25$ using sense primer $\mathrm{k} 11$ and antisense primer 352 as described above revealed three fragments in the patient (Fig. $2 A$ ), one of expected size (685 bp) and two shorter fragments ( $\sim 550$ and $316 \mathrm{bp}$ ). This finding was confirmed using a second set of primers (primers 296 and 297 described in Methods) which amplified a cDNA fragment extending from exons 21 to 26 (Fig. $2 \mathrm{~A}$ ). Further analysis of the 316-bp abnormal PCR product revealed a 369-bp in-frame

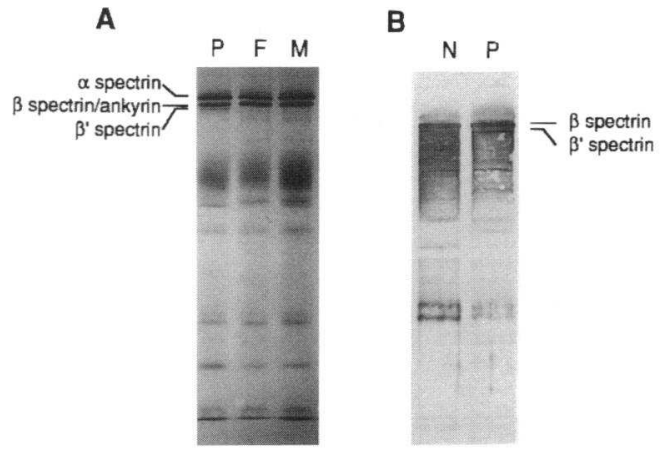

C

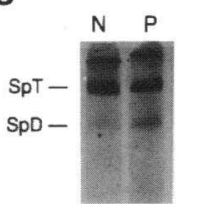

D

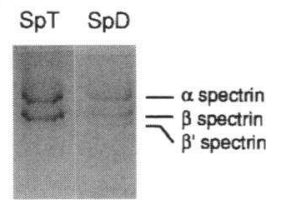

Figure 1. Biochemical analysis of the red cell membrane proteins. (A) SDS-PAGE analysis (10\% Laemmli gel) of the red cell membrane proteins of the patient $(P)$ and both parents $(F$ and $M$ ) revealing an extra band ( $\beta^{\prime}$ spectrin) migrating below the $\beta$ spectrin/ankyrin band in the patient; $(B)$ Western blot analysis of red cell membrane proteins separated on a $10 \%$ Laemmli gel, using an antibody directed against $\beta$ spectrin. Note the truncated band $\left(\beta^{\prime}\right.$ spectrin $)$ in the patient $(P)$ absent from the normal sample $(N)$; $(C)$ Electrophoresis under nondenaturing conditions of spectrin extracted from the membrane at $4^{\circ} \mathrm{C}$ showing a reproducible increase in the spectrin dimer to tetramer ratio in the patient $(P)$ (18\% spectrin dimer, $S p D)$ compared to the normal control $(N)$ $(<10 \%$ spectrin dimer $) ;(D)$ second dimension SDS-PAGE of spectrin dimers and tetramers separated in the first dimension under nondenaturing conditions at $4^{\circ} \mathrm{C}$. Note that the dimeric fraction is enriched in the truncated band compared to the tetrameric fraction.

deletion of the sequence normally contributed by exons 22 and 23 (Fig. $2 \mathrm{~B}$ ). The corresponding portion of the protein represents most of repeated segment 12 and the $\mathrm{NH}_{2}$-terminal portion of repeated segment 13 (Fig. $2 \mathrm{C}$ ). The intermediate size fragment $(\sim 550 \mathrm{bp})$ represents a heteroduplex of the normal and truncated DNA fragments.

To elucidate the underlying molecular defect, genomic DNA, prepared from the three family members, was analyzed by Southern blot hybridization using two restriction enzymes independently (EcoRI and XbaI) and a cDNA probe extending from exon 21 to exon 24 . With both restriction enzymes, we found an additional band of lower molecular weight in the affected family member, suggesting the occurrence of a 4.6-kb deletion within one of the two alleles in this patient (data not shown). Neither parents showed the same abnormality, suggesting a de novo mutation in the proband. To confirm this result, we amplified by PCR a genomic DNA segment extending from exon 21 to exon 24 using two pairs of primers independently: sense primer K11 with antisense primer 300 and sense primer 296 with antisense primer 300 (Fig. 3). As the size of the amplified fragment would exceed $8 \mathrm{~kb}$ in the normal allele, no PCR product was expected from this amplification using a normal individual genomic DNA. Indeed, no PCR product was obtained from the normal control $(C)$. However, in the patient, this amplification yielded bands of $\sim 710$ and $\sim 650$ bp with the two sets of primers, respectively $(P)$. Sequencing of the 

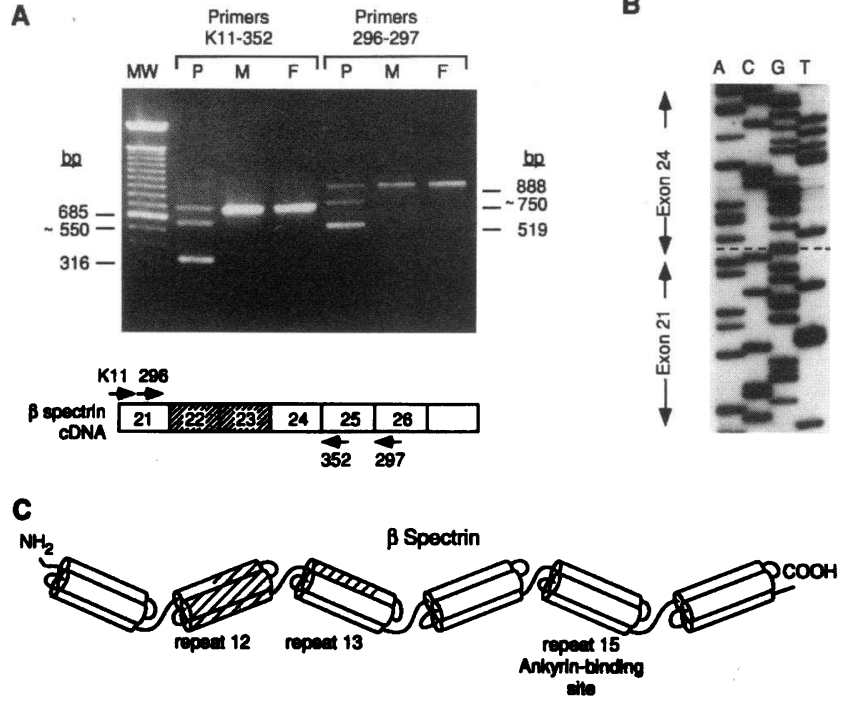

Figure 2. PCR amplification of reticulocyte $\beta$ spectrin cDNA. The sequences corresponding to exons $21-25$ and $21-26$ of the $\beta$ spectrin cDNA were amplified in the three family members using two sets of primers, respectively: primers K11 with 352 and primers 296 with 297 shown in the diagram. The first lane is the molecular weight marker $(M W)$. Note in both series of amplifications the fragment which is truncated by $369 \mathrm{bp}$ in the patient only $(P)$. The third band of intermediate size present in the patient represents a hybrid band between the normal and the truncated one. $(B)$ Subcloning and sequencing of the 685-bp fragment in the patient revealed the normal sequence corresponding to exon 21-25 of the $\beta$ spectrin cDNA (data not shown). However, the sequence corresponding to exons 22 and 23 were deleted from the 316-bp fragment as shown. This deletion of $369 \mathrm{bp}$ did not alter the reading frame. $(C)$ Schematic representation of $\beta$ spectrin showing the region deleted from the protein (shaded area). This corresponds to most of repeated segment 12 along with part of the first helix of repeated segment 13. The ankyrin-binding site has been localized to repeated segment 15 .

650-bp fragment revealed the normal sequence corresponding to exons 21 and 24 on either sides connected through normal boundaries to introns 21 and 23 , respectively. Therefore, the deletion includes part of intron 21 , exon 22 , intron 22 , exon 23 , and part of intron 23.

\section{Molecular basis for the spectrin deficiency}

To explain the imbalance between the amounts of the normal and the truncated $\beta$ spectrin present on the membrane, we have addressed several possibilities: $(a)$ instability and degradation of the transcriptional message, and $(b)$ defective synthesis, stability, or incorporation into the membrane of the truncated protein.

Quantitation of spectrin mRNA and stability. We first examined the stability of the mRNA by relative quantitation of cDNA as described in Methods. The PCR amplification of the patient's cDNA, using primers 296 and 297 flanking the deleted segment, yields two fragments, one of 888 bp corresponding to the normal allele and a 519-bp fragment corresponding to the mutant allele. The relative amount of these two products was examined after each PCR cycle between cycles 15 and 30 as described in Methods. We find that the ratio of the truncated to the normal band remains fairly constant around 1.4 (data not shown). This result suggests that the mRNA generated by the transcription of the mutant $\beta$ spectrin gene is stable and present at least in

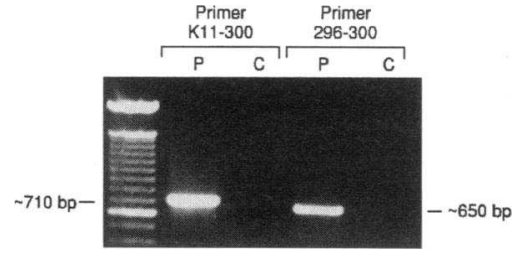

Figure 3. PCR amplification of $\beta$ spectrin genomic DNA. Using two sets of primers independently ( sense primer K11 with antisense primer 300 and sense primer 296 with antisense primer 300) shown on the dia-

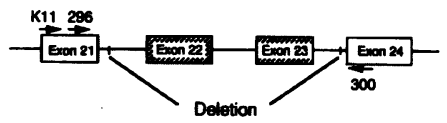
gram, PCR amplification of genomic DNA from the patient $(P)$ and a control sample $(C)$ was carried out. Bands of $\sim 710 \mathrm{bp}$ and $650 \mathrm{bp}$ were obtained with the two sets of primers, respectively, in the patient only. As expected, no product is seen in the control sample as the estimated sizes of the amplified fragments exceed $8 \mathrm{~kb}$ in the normal allele. Subcloning and sequencing of the 650-bp fragment revealed the normal sequence corresponding to exons 21 and 24 on either sides connected through normal boundaries to introns 21 and 23, respectively, confirming the deletion of part of intron 21 , exon 22, intron 22, exon 23 , and part of intron 23.

the same amount as the normal transcript in the cytoplasm of reticulocytes.

Synthesis, stability, and membrane incorporation of spectrin. After excluding instability of the mutant mRNA, we analyzed the protein synthesis using an in vitro liquid culture system allowing the isolation of late erythroblasts from peripheral blood BFU erythroblasts. Pulse label experiments described in Methods reveal that, in the erythroblast cytosolic fraction, the truncated $\beta$ spectrin is readily immunoprecipitated and is as abundant as the normal protein. Likewise, the high ratio of $\alpha$ spectrin to $\beta$ spectrin, indicating an excessive synthesis of $\alpha$ spectrin $(26,27)$, is unperturbed (Fig. $4 A$ ). However, these experiments also show that the truncated $\beta$ spectrin is virtually absent from the skeletal fraction (Fig. $4 A$ ). In this fraction, the $\alpha$ spectrin to $\beta$ spectrin ratio is one, reflecting the fact that, on the membrane, spectrin is present as heterodimers or higher order oligomers. The pulse chase experiments of the newly synthesized cytosolic spectrin show that at $0 \mathrm{~min}$ (i.e., at the

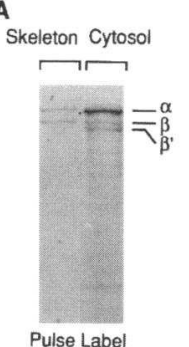

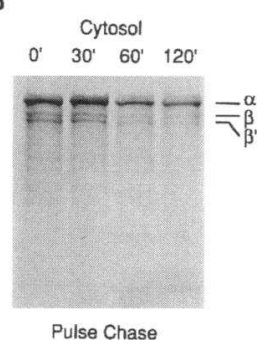

Figure 4. Synthesis, stability, and membrane incorporation of spectrin in the patient's erythroblasts. Using a liquid culture system, erythroblasts were obtained from peripheral blood BFU erythroblasts. $(A)$ In the pulse label experiment, erythroblasts were labeled for $90 \mathrm{~min}$ with $\left[{ }^{35} \mathrm{~S}\right.$ ] methionine before isolation of the skeletal and cytosolic fractions of the cells, followed by immunoprecipitation of spectrin. The autoradiogram shows that despite its normal presence in the cytosolic fraction, the truncated protein is almost absent from the skeletal fraction, suggesting a defective incorporation into the skeleton. $(B)$ In the pulse chase experiment, erythroblasts were labeled for 15 min. Further incorporation was stopped by the addition of unlabeled methionine. The cytosolic fraction was isolated and spectrin immunoprecipitated after incubation for different time intervals $(0,30,60$, and $120 \mathrm{~min}$ ). The autoradiogram shows that at $0 \mathrm{~min}$ (i.e., after $15 \mathrm{~min}$ of labeling), the normal and truncated $\beta$ spectrin are present in equal amounts and degrade with a similar rate over the ensuing $120 \mathrm{~min}$. 

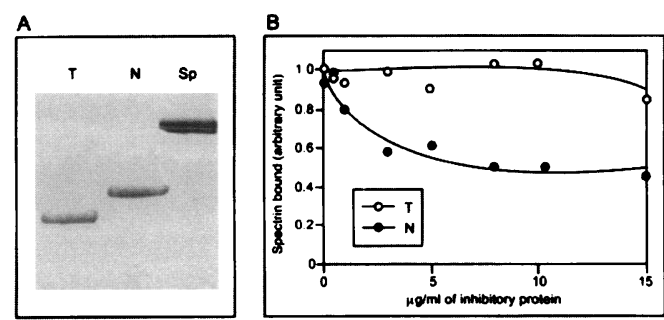

Figure 5. Binding of synthetic peptides to IOVs. (A) SDS-PAGE gel stained with Coomassie blue of purified spectrin extracts $(S p)$ and normal $(N)$ and truncated $(T)$ synthetic peptides. Both peptides were synthesized as described in Methods. They both extend from residues 13971898 of the $\beta$ spectrin molecule. (B) Typical inhibition curve of spectrin binding to IOVs by the synthetic peptides: the recombinant synthetic peptides were tested for their ability to competitively inhibit the binding of iodinated purified spectrin to IOVs. As shown, the normal protein $(\bullet)$ is capable of rapid and effective inhibition of the spectrin binding (with a maximal inhibition of $\sim 75 \%$ at high concentrations), while the truncated protein $(0)$ exhibits minimal inhibition within the same concentration range. This curve is representative of four independent experiments.

end of the 15-min labeling period), the normal and the truncated proteins are present in equal amounts in the cytoplasm of erythroblasts and that the rate of degradation of the mutant protein parallels that of its normal counterpart during the ensuing 120 min of the chasing period (Fig. $4 B$ ). Therefore, we can conclude from these experiments that, despite normal synthesis and stability in the cytoplasm of erythroblasts, the truncated $\beta$ spectrin is inadequately incorporated into the membrane skeleton.

Defective binding of the mutant peptide to IOVs. Because of the proximity of the $\beta$ spectrin deletion to the ankyrin-binding domain located within repeated segment 15 , we postulated that the binding of the mutant spectrin to ankyrin may be affected. To test this hypothesis, we expressed in $E$. coli as described in Methods, two synthetic peptides extending from residue $1397-$ 1898 , one corresponding to the normal $\beta$ spectrin sequence, and the other containing the deleted portion (Fig. $5 \mathrm{~A}$ ). These two peptides contain repeated segments 15 and 16 , where the ankyrin-binding domain of spectrin has been localized. They both react with antibodies directed against $\beta$ spectrin. The choice of these specific peptides (i.e., from residue 1397 to 1898) was based on the extensive data available regarding the binding of the normal peptide to IOVs (39). We analyzed the competitive inhibition of the binding of iodinated, purified spectrin dimers to IOVs by each of the two synthetic peptides. In support of the proposed model, we find that the normal peptide is capable of rapid and effective inhibition of spectrin binding to IOVs (with a maximum inhibition of $75 \%$ at high concentrations), while the truncated peptide is only capable of minimal inhibition at the same concentrations (Fig. $5 \mathrm{~B}$ ). This result shows that the binding of the synthetic peptide to IOVs is affected by the presence of the deletion described in this paper and supports the model which is proposed that attributes the spectrin deficiency on the membrane to a defective binding of the mutant spectrin to ankyrin.

\section{Discussion}

To date, only one mutation of spectrin has been reported in association with HS (25). This mutant $\beta$ spectrin exhibited a defective binding to protein 4.1 , but the relation between the reported $\mathrm{NH}_{2}$ terminal mutation and the spectrin deficiency is unclear. Here, we describe a second mutant spectrin underlying a phenotype of HS with partial spectrin deficiency. The mutant protein is characterized by a truncated $\beta$ chain resulting from a large genomic deletion which includes exons 22 and 23 of the $\beta$ spectrin gene. The resulting mutant $\beta$ spectrin protein lacks 123 amino acids (residues 1492-1615). The normal $\beta$ spectrin can be divided into three structural domains (42). The middle domain, or domain II, consists of 17 homologous repeated segments and contains the ankyrin-binding site of $\beta$ spectrin localized to the 15 th repeated segment (39). The deletion described in this paper constitutes most of the 12th repeated segment along with one-third of the adjacent 13th repeated segment.

We find that, despite its normal synthesis and stability in the cytoplasm of erythroblasts, the truncated protein is inefficiently incorporated into the erythroblast skeleton. We postulated that this aberrancy in the vicinity of the ankyrin-binding site produces conformational changes of $\beta$ spectrin that may affect the binding of the abnormal heterodimer to the membrane or, considerably less likely, may affect the efficiency of the truncated protein to associate with $\alpha$ monomers to form spectrin heterodimers. We have provided evidence, based on synthetic peptide-binding assays, that the former mechanism may indeed be implicated in the spectrin deficiency.

Sequence analysis has previously suggested a model for the repetitive segments of spectrin which consists of the folding of each repetitive segment into a three helix bundle (3). More recent analysis of crystallized fragments of $\alpha$ spectrin has demonstrated the validity of this model and shown that the repetitive segments of spectrin preserve a fixed register between the third helix of one segment and the first helix of the adjacent segment (43). Interactions between residues at the boundary of these two helices and residues within the second helix restrict the segment to segment flexibility and predict that each segment is axially rotated $60^{\circ}$ with respect to the previous one. The deletion described in this paper markedly disrupts repeated segments 12 and 13. Moreover, this deletion includes on both segments, the tryptophan at position 45 , one of the two most invariant among the conserved residues throughout $\beta$ spectrin. Therefore, this truncation would be expected to significantly upset the rigid phasing which characterizes the normal $\beta$ spectrin, leading to a different conformation that may affect the ankyrin-binding domain of $\beta$ spectrin and, additionally, may prevent the $\alpha$ spectrin $-\beta$ spectrin heterodimer association.

The structural determinants of the ankyrin-binding domain of $\beta$ spectrin have recently been defined (39). Sequential carboxy-terminal and amino-terminal truncations of recombinant $\beta$ spectrin peptides enabled to localize the ankyrin-binding domain of $\beta$ spectrin to the 15 th repeated unit. It is easily conceivable that the deletion described in this paper and involving repeated units 12 and 13 results in a conformational change of the protein which affects the ankyrin-binding site due to its close proximity. Similar relatively long-range effects have been described in the spectrin molecule. Several mutations located within the second, third, fourth, and even fifth repeated segments of $\alpha$ spectrin have been described and were shown to affect the heterodimer self-association site located within the first triple helical unit (reviewed in reference 44). In these cases, this effect is believed to occur through conformational changes of the protein. This model, which is supported by the 
synthetic peptide-binding assays, could readily explain the misincorporation of the abnormal heterodimer into the skeleton through a defective binding of the mutant protein to ankyrin.

In addition, the side-to-side association of the two subunits of spectrin in an antiparallel orientation results from very strong interactions between the two molecules which, in vitro, occur within seconds $(45,46)$. Multiple subunit peptides involved in noncovalent associations between the two chains have been identified $(46,47)$. Recently, it was proposed that the heterodimer assembly may be initiated at a single, discrete region on each subunit directly opposite of each other (46). The unique conformation of the repeated segments involved in this nucleation site (in the case of $\beta$ spectrin, repeated segments 1-4) would initiate the association and determine the phasing of the subsequent segments interactions. In spectrin Durham, a longrange conformational modification affecting the nucleation site on the $\beta$ subunit could result in a marked decrease in its affinity for the complementary segment on the $\alpha$ subunit. Alternatively, it is conceivable that despite a normal initiation of the $\alpha / \beta$ subunit binding through the nucleation site the propagation of this binding might be offset by the disruption in the normal phasing of the $\beta$ spectrin repeats. The inefficient heterodimer formation could also lead, in this model, to further misincorporation of the truncated protein into the membrane skeleton.

The deletion described in this paper also affects the spectrin heterodimer self-association site. This is suggested by the increase in the dimer to tetramer ratio on the membrane as assessed by the nondenaturing gel electrophoresis of spectrin extracted at $4^{\circ} \mathrm{C}$, as well as by the finding that the dimeric fraction is enriched in the truncated $\beta$ spectrin. Such horizontal defect is characteristically associated with the phenotype of hereditary elliptocytosis (48). We postulate that this defect is overshadowed by the relatively severe overall spectrin deficiency characteristic of the HS phenotype.

It is not surprising that a heterozygous defect of $\beta$ spectrin would result in expression of the abnormal phenotype. It has been previously shown that the rate of synthesis of $\alpha$ spectrin is three times that of $\beta$ spectrin, and therefore the availability of $\beta$ spectrin determines the rate of assembly of the spectrin heterodimers on the membrane $(26,27)$. Supporting this concept is the previously reported $\beta$ spectrin mutation associated with HS (spectrin Kissimmee [25]) that was shown to have an autosomal dominant pattern of inheritance. It is interesting to note that, like in the spectrin Kissimmee mutation, the red cell phenotype described here is characterized by the presence of acanthocytes.

In summary, we describe a novel truncated $\beta$ spectrin associated with hereditary spherocytosis. We propose a new mechanism underlying the spectrin deficiency in HS which is based on a dysfunctional incorporation of the abnormal $\beta$ spectrin into the skeleton due to its defective binding to ankyrin.

\section{Acknowledgments}

We are indebted to Donna Marie Mironchuk for preparing the photographic illustrations.

This work was supported by grants from the National Institutes of Health No. K08 HL-02720 (to H. Hassoun) and HL-37462 and HL27215 (to J. Palek).

\section{References}

1. Palek, J., and K. E. Sahr. 1992. Mutations of the red blood cell membrane proteins: from clinical evaluations to detection of the underlying genetic defect. Blood. 80:308-330.
2. Bennett, V., and D. M. Gilligan. 1993. The spectrin-based membrane skeleton and micron-scale organization of the plasma membrane. Annu. Rev. Cell Biol. 9:27-66.

3. Speicher, D. W., and V. T. Marchesi. 1984. Erythrocyte spectrin is comprised of many homologous triple helical segments. Nature (Lond.). 311:177180.

4. Speicher, D. W., J. S. Morrow, W. J. Knowles, and V. T. Marchesi. 1982. A structural model of human erythrocyte spectrin. Alignment of chemical and functional domains. J. Biol. Chem. 257:9093-9101.

5. Shotton, D. M., B. E. Burke, and D. Branton. 1979. The molecular structure of human erythrocyte spectrin. Biophysical and electron microscopic studies. $J$. Mol. Biol. 131:303-320.

6. Morrow, J. S., and V. T. Marchesi. 1981. Self-assembly of spectrin oligomers in vitro: a basis for a dynamic cytoskeleton. J. Cell Biol. 88:463-468.

7. Agre, P., E. P. Orringer, and V. Bennett. 1982. Deficient red-cell spectrin in severe, recessively inherited spherocytosis. N. Engl. J. Med. 306:1155-1161.

8. Agre, P., A. Asimos, J. F. Casella, and D. McMillan. 1986. Inheritance pattern and clinical response to splenectomy as a reflection of erythrocyte spectrin deficiency in hereditary spherocytosis. N. Engl. J. Med. 315:1579-1583.

9. Agre, P., J. F. Casella, and W. H. Zinkham. 1985. Partial deficiency of erythrocyte spectrin in hereditary spherocytosis. Nature (Lond.). 314:380-383.

10. Coetzer, T. L., J. Lawler, S. C. Liu, J. T. Prchal, R. J. Gualtieri, M. C. Brain, J. V. Dacie, and J. Palek. 1988. Partial ankyrin and spectrin deficiency in severe, atypical hereditary spherocytosis. N. Engl. J. Med. 318:230-234.

11. Savvides, P., O. Shalev, K. M. John, and S. E. Lux. 1993. Combined spectrin and ankyrin deficiency is common in autosomal dominant hereditary spherocytosis. Blood. 82:2953-2957.

12. Iolascon, A., E. M. Giudice, C. Camaschella, L. Pinto, B. Nobili, S. Perrotta, and S. Cutillo. 1991. Ankyrin deficiency in dominant hereditary spherocytosis: report of three cases. Br. J. Haematol. 78:551-554.

13. Jarolim, P., P. Ruff, T. L. Coetzer, J. T. Prchal, S. K. Ballas, M. Poon, V. Barbec, and J. Palek. 1990. A subset of patients with dominantly inherited hereditary spherocytosis has a marked deficiency of the band 3 protein. Blood. 76(Suppl. 1):37a. (Abstr.)

14. Lux, S., C. Bedrosian, O. Shalev, M. Morris, J. Chasis, K. Davies, P. Savvides, and M. Telen. 1990. Deficiency of band 3 in dominant hereditary spherocytosis with normal spectrin content. Clin. Res. 38:300a. (Abstr.)

15. Miraglia del Giudice, E., S. Perrotta, L. Pinto, M. D. Cappellini, G. Fiorelli, S. Cutillo, and A. Iolascon. 1992. Hereditary spherocytosis characterized by increased spectrin/band 3 ratio. Br. J. Haematol. 80:133-136.

16. Saad, S. T. O., S. C. Liu, D. Golan, J. B. Corbett, H. S. Thatte, L. Derick, M. Hanspal, P. Jarolim, Fibach, and J. Palek. 1991. Mechanism underlying band 3 deficiency in a subset of patients with hereditary spherocytosis (HS). Blood. 78(Suppl. 1):81a. (Abstr.)

17. Ghanem, A., B. Pothier, J. Maréchal, M. T. Ducluzeau, L. Morlé, N. Alloisio, C. Féo, B. A. Abdeladhim, S. Fattoum, and J. Delaunay. 1990. A haemolytic syndrome associated with the complete absence of red cell membrane protein 4.2 in two Tunisian siblings. Br. J. Haematol. 75:414-420.

18. Ideguchi, H., J. Nishimura, H. Nawata, and N. Hamasaki. 1990. A genetic defect of erythrocyte band 4.2 protein associated with hereditary spherocytosis. Br. J. Haematol. 74:347-353.

19. Rybicki, A. C., R. Heath, J. L. Wolf, B. Lubin, and R. S. Schwartz. 1988 Deficiency of protein 4.2 in erythrocytes from a patient with a Coombs negative hemolytic anemia. J. Clin. Invest. 81:893-901.

20. Rybicki, A. C., J. J. H. Qiu, S. Musto, N. L. Rosen, R. L. Nagel, and R. S. Schwartz. 1993. Human erythrocyte protein 4.2 deficiency associated with hemolytic anemia and a homozygous ${ }^{40}$ glutamic acid $\rightarrow$ lysine substitution in the cytoplasmic domain of band 3 (band $3^{\text {Montefiore }}$ ). Blood. 81:2155-2165.

21. Jarolim, P., H. L. Rubin, S. Liu, M. R. Cho, V. Brabec, L. H. Derrick S. J. Yi, S. T. O. Saad, S. Alper, C. Brugnara, et al. 1994. Duplication of 10 nucleotides in the erythroid band 3 (AE1) gene is a kindred with hereditary spherocytosis and band 3 protein deficiency (band $3^{\text {PRAGUE }}$ ). J. Clin. Invest. 93:121-130.

22. Jarolim, P., H. L. Rubin, V. Brabec, and J. Palek. 1993. Abnormal alternative splicing of erythroid ankyrin mRNA in two kindred with hereditary spherocytosis (ankyrin ${ }^{\text {PRAGUE }}$ and ankyrin ${ }^{\text {RAKOVNIK }}$ ). Blood. 82(Suppl. 1):5a. (Abstr.)

23. Eber, S. W., M. L. Lux, J. M. Gonzalez, A. Scarpa, W. T. Tse, P. G. Gallagher, A. Pekrun, B. G. Forget, and S. E. Lux. 1993. Discovery of 8 ankyrin mutations in hereditary spherocytosis (HS) indicates that ankyrin defects are a major cause of dominant and recessive HS. Blood. 82 (Suppl. 1):3089a. (Abstr.)

24. Becker, P. S., W. T. Tse, S. E. Lux, and B. G. Forget. 1990. Identification of the molecular defect of $\beta$ spectrin in autosomal dominant hereditary spherocytosis (HS) associated with defective binding of protein 4.1, $\mathrm{HS}(S p-4.1)$. Blood. 76(Suppl. 1):25a. (Abstr.)

25. Becker, P. S., W. T. Tse, S. E. Lux, and B. G. Forget. 1993. $\beta$ Spectrin Kissimmee: a spectrin variant associated with autosomal dominant hereditary spherocytosis and defective binding to protein 4.1. J. Clin. Invest. 92:612-616. 26. Hanspal, M., and J. Palek. 1987. Synthesis and assembly of membrane keletal proteins in mammalian red cell precursors. J. Cell Biol. 105:1417-1424. 27. Hanspal, M., J. Hanspal, and R. Kalraiya. 1992. Asynchronous synthesis 
of membrane skeletal proteins during terminal maturation of murine erythroblasts. Blood. 80:530-539.

28. Coetzer, T. L., and J. Palek. 1986. Partial spectrin deficiency in hereditary pyropoikilocytosis. Blood. 67:919-924.

29. Dodge, J. T., C. Mitchell, and D. J. Hanahan. 1963. The preparation and chemical characteristics of hemoglobin-free ghosts of human erythrocytes. Arch. Biochem. Biophys. 100:119-130.

30. Laemmli, U. K. 1970. Cleavage of structural proteins during the assembly of the head of bacteriophage T4. Nature (Lond.). 227:680-685.

31. Liu, S. C., J. Palek, J. T. Prchal, and P. Castleberry. 1981. Altered spectrin dimer-dimer association and instability of erythrocyte membrane skeletons in hereditary pyropoikilocytosis. J. Clin. Invest. 68:597-605.

32. Goossens, M., and Y. W. Kan. 1981. DNA analysis in the diagnosis of hemoglobin disorders. Methods Enzymol. 76:805-809.

33. Sykes, B. J. 1983. DNA in heritable diseases. Lancet. ii:787-788.

34. Sambrook, J., E. F. Fritsch, and T. Maniatis. 1989. Molecular Cloning: A Laboratory Manual. Cold Spring Harbor Laboratory, Cold Spring Harbor, NY. 8.

35. Sanger, F., S. Nicklen, and A. R. Coulson. 1977. DNA sequencing with chain termination inhibitors. Proc. Natl. Acad. Sci. USA. 74:5463-5467.

36. Hanspal, M., J. S. Hanspal, K. E. Sahr, E. Fibach, J. Nachman, and J. Palek. 1993. Molecular basis of spectrin deficiency in hereditary pyropoikilocytosis. Blood. 82:1652-1660.

37. Fibach, E., D. Manor, A. Oppenheim, and E. A. Rachmilewitz. 1989. Proliferation and maturation of human erythroid progenitors in liquid culture. Blood. 73:100-106.

38. Frangioni, J. V., and B. G. Neel. 1993. Solubilization and purification of enzymatically active glutathione S-transferase (pGEX) fusion proteins. Anal. Biochem. 210:179-187.

39. Kennedy, S. P., S. L. Warren, B. G. Forget, and J. S. Morrow. 1991.
Ankyrin binds to the 15 th repetitive unit of erythroid and nonerythroid $\beta$-spectrin J. Cell Biol. 115:267-277.

40. Bennett, V., and P. J. Stenbuck. 1979. Identification and partial purification of ankyrin, the high affinity membrane attachment site for human erythrocyte spectrin. J. Biol. Chem. 254:2533-2541.

41. Bennett, V., and D. Branton. 1977. Selective association of spectrin with the cytoplasmic surface of human erythrocyte plasma membranes. J. Biol. Chem. 252:2753-2763.

42. Winkelmann, J. C., J. C. Chang, W. T. Tse, A. L. Scarpa, V. T. Marchesi, and B. G. Forget. 1990. Full length sequence of the cDNA for human erythroid $\beta$ spectrin. J. Biol. Chem. 265:11827-11832.

43. Yan, Y., E. Winograd, T. Viel, T. Cronin, S. C. Harrison, and D. Branton. 1993. Crystal structure of the repetitive segments of spectrin. Science (Wash DC). 262:2027-2030.

44. Delaunay, J., and D. Dhermy. 1993. Mutations involving the spectrin heterodimer contact site: clinical expression and alterations in specific function. Semin. Hematol. 30:21-33.

45. Yoshino, H., and V. T. Marchesi. 1984. Isolation of spectrin subunits and reassociation in vitro. Analysis by fluorescence polarization. J. Biol. Chem. 259:4496-4500.

46. Speicher, D. W., L. Weglarz, and T. M. DeSilva. 1992. Properties of human red cell spectrin heterodimer (side-to-side) assembly and identification of an essential nucleation site. J. Biol. Chem. 267:14775-14782.

47. Morrow, J. S., D. W. Speicher, W. J. Knowles, J. Hsu, and V. T. Marchesi. 1980. Identification of functional domains of human erythrocyte spectrin (peptide maps/cytoskeleton/membrane binding/peptide association/protein structure). Proc. Natl. Acad. Sci. USA. 77:6592-6596.

48. Palek, J. 1987. Hereditary elliptocytosis, spherocytosis and related disorders: consequences of a deficiency or a mutation of membrane skeletal proteins. Blood Rev. 1:147-168. 